$$
\begin{array}{r}
\text { Pontifícia Universidade Católica } \\
\text { Do Rio de Janeiro }
\end{array}
$$

Bruno Francisco Teixeira Simões

\title{
Controle Estatístico de Processos Multicanal
}

Tese apresentada ao Programa de Pós-graduação em Engenharia de Produção do Departamento de Engenharia Industrial da PUC-Rio como parte dos requisitos parciais para obtenção do título de Doutor em Engenharia de Produção

Orientador: Prof. Eugenio Kahn Epprecht 


\section{Bruno Francisco Teixeira Simões}

\section{Controle Estatístico de Processos Multicanal}

Tese apresentada como requisito parcial para obtenção do grau de Doutor pelo Programa de Pós-Graduação em Engenharia de Produção da PUC-Rio. Aprovada pela Comissão Examinadora abaixo assinada.

Prof. Eugenio Kahn Epprecht

Orientador

Departamento de Engenharia Industrial - PUC-Rio

Prof. Flávio Sanson Fogliatto

UFRGS

Prof. Annibal Parracho Sant'Anna

UFF

Prof. Antonio Fernando de Castro Vieira Departamento de Engenharia Industrial - PUC-Rio

Prof. Reinaldo Castro Souza

Departamento de Engenharia Elétrica - PUC-Rio

Prof. José Eugenio Leal

Coordenador Setorial do Centro

Técnico Científico - PUC-Rio

Rio de Janeiro, 31 de maio de 2010 
Todos os direitos reservados. É proibida a reprodução total ou parcial do trabalho sem autorização da universidade, do autor e do orientador.

\section{Bruno Francisco Teixeira Simões}

Graduou-se em Estatística pela UERJ (Universidade do Estado do Rio de Janeiro) no início de 2004. Obteve o título de Mestre em Engenharia de Produção (área de concentração: Gerência de Produção) pela Pontifícia Universidade Católica do Rio de Janeiro (PUC-Rio) com a dissertação intitulada "Gráfico EWMA com Constante de Amortecimento Adaptativa para Controle Estatístico de Processos” em 2006. Desde então tem atuado na linha de pesquisa de Engenharia da Qualidade, com artigos aceitos em bons periódicos internacionais.

Ficha Catalográfica

Simões, Bruno Francisco Teixeira

Controle estatístico de processos multicanal / Bruno Francisco Teixeira Simões ; orientador: Eugenio Kahn Epprecht. - 2010.

259 f. : il. ; $30 \mathrm{~cm}$

Tese (Doutorado em Engenharia de Produção) Pontifícia Universidade Católica do Rio de Janeiro, Rio de Janeiro, 2010.

Inclui referências bibliográficas

1. Engenharia Industrial - Teses. 2. Gráficos de controle de grupos. 3. Processos multicanal. 4. Média móvel exponencialmente ponderada. 5. Média.6. Variância. I. Eugenio Kahn Epprecht. II. Pontifícia Universidade Católica do Rio de Janeiro. Departamento de Engenharia Industrial. III. Título. 
Para meus pais Ana Maria e José Francisco pelo amor e dedicação 


\section{Agradecimentos}

O meu eterno agradecimento a Deus, para Ele toda a honra e toda a glória. A minha gratidão pelo dom da vida e caminho abençoado pelo seu infinito amor e a sua misericórdia, me direcionando sempre o melhor e, por me colocar próximo a pessoas altamente capacitadas e de boa vontade, tornando-me hábil a desenvolver o meu potencial.

Aos meus pais, Ana Maria e José Francisco, pelo amor, incentivo, participação e a excelente estrutura familiar, junto aos meus queridos irmãos.

Ao orientador e amigo Professor Doutor Eugenio Kahn Epprecht pela excelente orientação, por acreditar em mim e no meu trabalho, pela lealdade e dedicação, pelo seu incentivo e sua ponderação, tornando sempre possível a boa realização de pesquisas de qualidade e relevância no meio científico.

Aos professores que participaram da Comissão Examinadora desta tese, do exame de qualificação e de proposta de tese, que contribuíram com conselhos preciosos para a elaboração deste trabalho.

Aos funcionários e professores do Departamento de Engenharia Industrial que, no cumprimento do seu dever, sempre me trataram com cordialidade e boa vontade.

À PUC-Rio que, por intermédio do orientador Professor Doutor Eugenio Kahn Epprecht, me forneceu a estrutura acadêmica.

Ao CNPq pelo apoio financeiro concedido, com o qual foi possível a realização dos meus estudos de pós-graduação. 


\section{Resumo}

Simões, Bruno Francisco Teixeira; Epprecht, Eugenio Kahn (Orientador). Controle Estatístico de Processos Multicanal. Rio de Janeiro, 2010. 259p. Tese de Doutorado - Departamento de Engenharia Industrial, Pontifícia Universidade Católica do Rio de Janeiro.

Processos Multicanal (PMC) estão presentes nas linhas de produção de muitos segmentos industriais, tais como na indústria alimentícia, farmacêutica, de fabricação de aço e de papel. No entanto, há poucos trabalhos na literatura dedicados ao controle estatístico de processos dessa natureza. O trabalho de Boyd (1950) é o primeiro deles. Neste trabalho são descritos os gráficos de controle de grupos (GCG). Este é o procedimento tradicional, recomendado em textos didáticos de CEP como Pyzdek (1992) e Montgomery (até a 3a edição, de 1997). Posteriormente, Mortell e Runger (1995) elaboram um modelo matemático mais realista para PMC, decompondo a fonte de variação do processo em duas componentes distintas: uma, comum a todos os canais e outra, correspondendo à variação individual de cada canal do processo. Tal modelo foi tão bem aceito na literatura que, desde a sua publicação, tem sido utilizado para o desenvolvimento de esquemas de controle mais eficientes para PMC. Dos esquemas desenvolvidos na versão Shewhart, para o controle estatístico das médias das componentes individuais de variação, devem ser destacados os gráficos de controle de Mortell e Runger (1995), de Runger, Alt e Montgomery (1996) e o GCG de Barbosa (2008). Dentre os esquemas mencionados, somente os dois primeiros foram desenvolvidos tanto em uma versão "de Shewhart" como em uma versão EWMA (Exponentially Weighted Moving Average), visando obter maior sensibilidade a pequenas alterações na média. Esta tese traz novas propostas para PMC bem representados pelo modelo de Mortell e Runger (1995). Propõe-se a análise da eficiência dos gráficos de controle existentes na detecção de aumentos na dispersão de um canal, bem como o desenvolvimento, na versão Shewhart e EWMA, de novos GCG especificamente destinados à sinalização de tais aumentos. Quando não é viável obter mais de uma observação por canal do processo, propõem-se os gráficos: GCG de MR das diferenças em relação ao nível-base (DNB) e GCG EWMA MR DNB. Já para as situações em que é possível obter mais de uma observação por canal, propõem-se: GCG de $S^{2}$ e GCG EWMA de $\ln \left(\mathrm{S}^{2}\right)$. É importante ressaltar que todos os trabalhos desenvolvidos na literatura (seguindo o modelo de Mortell e Runger, 1995) foram dedicados exclusivamente ao controle estatístico da média das componentes individuais de variação, portanto, esta tese tem caráter inédito. Além das contribuições mencionadas, visando obter maior sensibilidade a alterações de pequena magnitude na média das componentes individuais, propõe-se e analisa-se uma versão EWMA do GCG de Barbosa (2008), o mais eficiente na versão Shewhart. Adicionalmente, para obter esquemas EWMA mais eficientes, são obtidos os projetos ótimos de todos os esquemas EWMA apresentados nesta tese, incluindo os gráficos de controle de EWMA de $\mathrm{R}_{\mathrm{t}}$ de Mortell e Runger (1995) e de MEWMA de $S^{2}$ de Runger, Alt e Montgomery (1996). São analisadas as curvas de desempenho de todos os esquemas de controle para uma variedade de situações. Nas análises de desempenho, pode-se observar que os esquemas propostos nesta tese são os mais eficientes.

\section{Palavras-chave}

Gráficos de controle de Grupos; Processos Multicanal; Média Móvel Exponencialmente Ponderada; Média; Variância. 


\section{Abstract}

Simões, Bruno Francisco Teixeira; Epprecht, Eugenio Kahn (Advisor). Statistical Control of Multiple Stream Process. Rio de Janeiro, 2010. 259p. DSc. Thesis - Departamento de Engenharia Industrial, Pontifícia Universidade Católica do Rio de Janeiro.

In a multiple stream process (MSP), a same quality variable is measured in several streams in parallel. The first tool proposed for monitoring MSPs was the Group Control Chart (GCC) by Boyd (1950). These schemes are recommended in textbooks and guides as Pyzdek (1992) and Montgomery (until 3rd edition, 1997). Its efficiency is impaired by the presence of cross correlation between streams. A useful model for MSPs (Mortell and Runger, 1995) represents the value of the quality variable in each stream at any time $t$ as the sum of a random variable (or stochastic process) but that is common to all streams, which can be called base level, plus the individual variation of each stream relative to the base level. In the literature, three different Shewhart schemes were developed to control the individual variation of each stream: Mortell e Runger (1995), Runger, Alt and Montgomery (1996) and Barbosa (2008). Only the two first ones were developed both in a Shewhart-type and a EWMA (Exponentially Weighted Moving Average) version. All these schemes were devoted to monitoring the mean of the individual components of the streams; to the best of our knowledge, no previous work considered the case of increases in the variance of a stream. In this thesis four different GCCs for monitoring the inner variability of the individual streams are developed: a GCC of $\mathrm{S}^{2}$, the sample variance of each stream (which is not the same as Runger, Alt and Montgomery's statistics); a GCC of EWMA(lnS ${ }^{2}$ ); a GCC of the Moving Ranges of the residuals of each stream to the estimated base level, and an EWMA version of it. The last two GCCs cater for the case where, at every sampling time, only individual observations per stream are feasible, which is frequent with a large number of streams. Beyond the mentioned contributions, aiming at more sensitivity to the small shifts in the mean of the individual components, this work proposes a EWMA version of the GCC by Barbosa (2008), the most efficient in the Shewhart version. The ARL performance of every one of these schemes is analyzed, in a variety of situations, including the case of increases in the variance of one stream when the schemes are designed for monitoring the means of individual streams. The results show that the proposed schemes are the fastest in detecting special causes that affect one individual stream.

\section{Keywords}

Group Control Chart; Multiple Stream Process; Exponentially weighted moving average; Mean; Variance. 


\section{Sumário}

1. Introdução 23

1.1. Objetivos da Tese 28

1.2. Contribuições científicas e originalidade 29

1.3. Estrutura da Tese 29

2. Fundamentação Teórica e Trabalhos Precedentes 32

2.1. O Esquema EWMA 33

2.2. Trabalhos Precedentes - Visão Geral 38

2.3. Os Gráficos de Controle de Boyd (1950) 47

2.4. A proposta de Mortell e Runger (1995) 52

2.4.1. Gráfico de $R_{t}$ tipo Shewhart 54

2.4.2. Gráfico de controle em estilo EWMA 57

2.5. A proposta de Runger, Alt e Montgomery (1996) 58

2.5.1. Gráfico de controle de $\mathrm{T}^{2}$ das $\mathrm{c}-1$ componentes remanescentes

2.5.2. Gráfico de controle MEWMA das c-1 componentes remanescentes

2.6. A proposta de Barbosa (2008) 66

3. Controle Estatístico da Dispersão 74

3.1. Estudo analítico das medidas de desempenho do GCG das diferenças no caso de aumentos da dispersão de um canal

3.2. Gráficos específicos para o controle da dispersão

3.2.1. GCG versão Shewhart

84

3.2.1.1. Dados subgrupados: GCG de $S^{2} \quad 86$

3.2.1.2. Observações individuais: GCG de MR 87

3.2.2. GCG versão EWMA 89

3.2.2.1. Dados subgrupados: GCG de EWMA de $\ln \left(S^{2}\right)$

3.2.2.2. Observações individuais: GCG de EWMA de MR 94

4. Análise de Desempenho dos Gráficos tipo Shewhart para o Controle Estatístico da Dispersão $\quad 97$

4.1. Eventos de Interesse do GCG das Diferenças 97

4.2. Medidas de Desempenho 98

4.3. Procedimentos de Simulação 100

4.4. Resultados de Desempenho Individual 105

4.4.1. Controle por Observações Individuais 105

4.4.2. Controle por Dados Subgrupados 112

4.5. Resultados de Desempenho Conjunto 119

4.5.1. Controle por Observações Individuais 119

4.5.2. Controle por Dados Subgrupados 122

4.6. Cálculo Probabilístico de Eventos 126 
5. Controle Estatístico da Média 131

5.1. A estatística EWMA das diferenças 131

5.2. Os limites de controle do GCG de EWMA DNB 132

6. Análise de Desempenho dos Gráficos EWMA para o Controle Estatístico da Média

6.1. Medidas de desempenho 134

6.2. Procedimentos de Simulação 135

6.2.1. Primeira Parte da Corrida de Simulação: Período de Aquecimento da Estatística EWMA 136

6.2.2. Segunda Parte da Corrida: Cálculo do $\mathrm{NMA}_{1}$

6.3. Obtenção dos Projetos Ótimos 141

6.3.1. Curvas de isoNMA ${ }_{0}$

6.3.2. Projetos ótimos 145

6.4. Resultados de Desempenho 154

6.4.1. Controle por Observações Individuais 154

6.4.2. Controle por Dados Subgrupados 162

6.5. GCG de EWMA DNB: detecção do canal afetado 169

7. Análise de Desempenho dos Gráficos EWMA para o Controle Estatístico da Dispersão

7.1. Medidas de desempenho 176

7.2. Procedimentos de Simulação 176

7.2.1. Período de Aquecimento da Estatística EWMA 177

7.2.2. Segunda Parte da Corrida: Cálculo do $\mathrm{NMA}_{1} \quad 179$

7.3. Obtenção dos Projetos Ótimos 183

$\begin{array}{ll}\text { 7.3.1. Curvas de isoNMA } & 183\end{array}$

7.3.2. Projetos ótimos 186

7.4. Resultados de Desempenho Individual 190

7.4.1. Controle por Observações Individuais 190

7.4.2. Controle por Dados Subgrupados 198

7.5. Resultados de Desempenho Conjunto 205

7.5.1. Controle por Observações Individuais 206

7.5.2. Controle por Dados Subgrupados 210

8. Conclusões e Sugestões para pesquisas futuras 214

8.1. Conclusões 214

8.2. Sugestões para pesquisas futuras 218

9. Referências bibliográficas 220

10. Apêndice A: Contribuições originais da tese 226

11. Apêndice B: Relações entre os parâmetros $\lambda$ e $K$ de cada gráfico de controle analisado

12. Apêndice C: Medidas de desempenho dos Gráficos de Controle de EWMA para o controle estatístico da média 
13. Apêndice D: Medidas de desempenho individual dos Gráficos de Controle de EWMA para o controle estatístico da dispersão

14. Apêndice $\mathrm{E}$ : Medidas de desempenho conjunto dos Gráficos de Controle de EWMA para o controle estatístico da dispersão 


\section{Lista de tabelas e quadros}

Quadro 2.1 - Trabalhos na literatura de PMC

Tabela 2.1 - Comprimento de corrida significativo para um único canal

Tabela 2.2 - Valores de $\mathrm{NMA}_{0}$ obtidos para diferentes comprimentos de corridas pelo critério de corridas proposto por Nelson (1986)

Tabela 2.3 - Correlação entre $\hat{e}_{t i}$ e $\hat{e}_{t j}$. (para $i \neq j$ )

Tabela 2.4 - Diferenças (\%) nos cálculo de $\mathrm{NMA}_{0}$ e valores de $\mathrm{K}$ para os valores de $\mathrm{NMA}_{0}$ especificados, considerando processos compostos por três canais $(c=3)$

Tabela 4.1 - Medidas de Desempenho Individual para o controle por observações individuais

Tabela 4.2 - Exemplos de valores de SDRL das medidas de desempenho individuais dos gráficos para o controle por observações individuais

Tabela 4.3 - Valores de $\mathrm{NMA}_{1}$ de Zero-State e Steady-State para o GCG de MR

Tabela 4.4 - Medidas de Desempenho Individual para o controle por amostras de tamanho igual a quatro

Tabela 4.5 - Exemplos de valores de SDRL das medidas de desempenho individuais dos gráficos para o controle por amostras de tamanho igual a quatro

Tabela 4.6 - Medidas de Desempenho Conjunto para o controle por observações individuais

Tabela 4.7 - Medidas de Desempenho Conjunto para o controle por amostras de tamanho igual a quatro

124

Tabela 4.8 - Cálculo Probabilístico dos Eventos AUO e O-A 129

Tabela 6.1 - Projetos ótimos para o GCG de EWMA DNB $(n=1) \quad 147$

Tabela 6.2 - Projetos ótimos para o GCG de EWMA DNB $(n=4) \quad 148$

Tabela 6.3 - Projetos ótimos para o GC de EWMA de $\mathrm{R}_{\mathrm{t}}(n=1) \quad 149$

Tabela 6.4 - Projetos ótimos para o GC de EWMA de $\mathrm{R}_{\mathrm{t}}(n=4) \quad 150$

Tabela 6.5 - Projetos ótimos para o GC de MEWMA de $S^{2}(n=1) \quad 151$

Tabela 6.6 - Projetos ótimos para o GC de MEWMA de $S^{2}(n=4) \quad 152$

Tabela 6.7 - Medidas de Desempenho para $n=1$ e $\mathrm{NMA}_{0}=200$ (Parte I)

Tabela 6.8 - Medidas de Desempenho para $n=1$ e $\mathrm{NMA}_{0}=200$ (Parte II)

Tabela 6.9 - Comparação entre os valores de $\mathrm{NMA}_{1}$ de steadystate e zero-state $(n=1)$

Tabela 6.10 - Medidas de Desempenho para $n=4$ e $\mathrm{NMA}_{0}=200$ (Parte I) 
Tabela 6.11 - Medidas de Desempenho para $n=4$ e $\mathrm{NMA}_{0}=200$ (Parte II)

Tabela 6.12 - Comparação entre os valores de $\mathrm{NMA}_{1}$ de steadystate e zero-state $(n=4)$

Tabela 6.13 - Valores de $\mathrm{NMA}_{1}$ de steady-state do evento A (canal afetado sinaliza) para o GCG de EWMA DNB $(n=1)$

Tabela 6.14 - Valores de $\mathrm{NMA}_{1}$ de steady-state do evento $\mathrm{A}$ (canal afetado sinaliza) para o GCG de EWMA DNB ( $n=4$

Tabela 7.1 - Projetos ótimos para o GCG de EWMA de MR de DNB $(n=1)$

Tabela 7.2 - Projetos ótimos para o GCG de EWMA de $\ln \left(S^{2}\right)$ $(n=4)$

Tabela 7.3 - Medidas de Desempenho Individual para $n=1$ e $\mathrm{NMA}_{0}=200$ (Parte I)

Tabela 7.4 - Medidas de Desempenho Individual para $n=1$ e $\mathrm{NMA}_{0}=200$ (Parte II)

Tabela 7.5 - Comparação entre os valores de $\mathrm{NMA}_{1}$ de steadystate e zero-state $(n=1)$

Tabela 7.6 - Medidas de Desempenho Individual para $n=4$ e $\mathrm{NMA}_{0}=200$ (Parte I)

Tabela 7.7 - Medidas de Desempenho Individual para $n=4$ e $\mathrm{NMA}_{0}=200$ (Parte II)

Tabela 7.8 - Comparação entre os valores de $\mathrm{NMA}_{1}$ de steadystate e zero-state $(n=4)$

Tabela 7.9 - Medidas de Desempenho Conjunto para $n=1$ e $\mathrm{NMA}_{0}=200$ (Parte I)

Tabela 7.10 - Medidas de Desempenho Conjunto para $n=1$ e $\mathrm{NMA}_{0}=200$ (Parte II)

Tabela 7.11 - Medidas de Desempenho Conjunto para $n=4$ e $\mathrm{NMA}_{0}=200$ (Parte I)

Tabela 7.12 - Medidas de Desempenho Conjunto para $n=4$ e $\mathrm{NMA}_{0}=200$ (Parte II)

Tabela 8.1 - Relação de gráficos de controle analisados nesta tese

Quadro 10.1 - Lista das Contribuições desta Tese

Tabela 11.1 - Relação $\lambda \times K$ para o GCG de EWMA das diferenças em relação ao nível-base

Tabela 11.2 - Relação $\lambda \times K$ para o Gráfico EWMA de $\mathrm{R}_{\mathrm{t}}$

Tabela 11.3 - Relação $\lambda \times K$ para o Gráfico MEWMA de $S^{2}$

Tabela 11.4 - Relação $\lambda \times K$ para o GCG de EWMA de $\ln \left(S^{2}\right)$

Tabela 11.5 - Relação $\lambda \times K$ para o GCG de EWMA de MR das diferenças em relação ao nível-base

Tabela 12.1 - Medidas de Desempenho para $n=1$ e $\mathrm{NMA}_{0}=100$ (Parte I)

Tabela 12.2 - Medidas de Desempenho para $n=1$ e $\mathrm{NMA}_{0}=100$ (Parte II)

Tabela 12.3 - Medidas de Desempenho para $n=1$ e $\mathrm{NMA}_{0}=370,4$ (Parte I)

Tabela 12.4 - Medidas de Desempenho para $n=1$ e $\mathrm{NMA}_{0}=370,4$ (Parte II) 
Tabela 12.5 - Medidas de Desempenho para $n=4$ e $\mathrm{NMA}_{0}=100$ (Parte I)

Tabela 12.6 - Medidas de Desempenho para $n=4$ e $\mathrm{NMA}_{0}=100$ (Parte II)

Tabela 12.7 - Medidas de Desempenho para $n=4$ e $\mathrm{NMA}_{0}=370,4$ (Parte I)

Tabela 12.8 - Medidas de Desempenho para $n=4$ e $\mathrm{NMA}_{0}=370,4$ (Parte II)

Tabela 13.1 - Medidas de Desempenho Individual para $n=1$ e $\mathrm{NMA}_{0}=100$ (Parte I)

Tabela 13.2 - Medidas de Desempenho Individual para $n=1$ e $\mathrm{NMA}_{0}=100$ (Parte II)
Tabela 13.3 - Medidas de Desempenho Individual para $n=1$ e $\mathrm{NMA}_{0}=370,40$ (Parte I)

Tabela 13.4 - Medidas de Desempenho Individual para $n=1$ e $\mathrm{NMA}_{0}=370,40$ (Parte II)

Tabela 13.5 - Medidas de Desempenho Individual para $n=4$ e $\mathrm{NMA}_{0}=100$ (Parte I)

Tabela 13.6 - Medidas de Desempenho Individual para $n=4$ e $\mathrm{NMA}_{0}=100$ (Parte II)

Tabela 13.7 - Medidas de Desempenho Individual para $n=4$ e $\mathrm{NMA}_{0}=370,40$ (Parte I)

Tabela 13.8 - Medidas de Desempenho Individual para $n=4$ e $\mathrm{NMA}_{0}=370,40$ (Parte II)

Tabela 14.1 - Medidas de Desempenho Conjunto para $n=1$ e $\mathrm{NMA}_{0}=100$ (Parte I)

Tabela 14.2 - Medidas de Desempenho Conjunto para $n=1$ e $\mathrm{NMA}_{0}=100$ (Parte II)

Tabela 14.3 - Medidas de Desempenho Conjunto para $n=1$ e $\mathrm{NMA}_{0}=370,40$ (Parte I)

Tabela 14.4 - Medidas de Desempenho Conjunto para $n=1$ e $\mathrm{NMA}_{0}=370,40$ (Parte II)

Tabela 14.5 - Medidas de Desempenho Conjunto para $n=4$ e $\mathrm{NMA}_{0}=100$ (Parte I)

Tabela 14.6 - Medidas de Desempenho Conjunto para $n=4$ e $\mathrm{NMA}_{0}=100$ (Parte II)

Tabela 14.7 - Medidas de Desempenho Conjunto para $n=4$ e $\mathrm{NMA}_{0}=370,40$ (Parte I)

Tabela 14.8 - Medidas de Desempenho Conjunto para $n=4$ e $\mathrm{NMA}_{0}=370,40$ (Parte II) 


\section{Lista de figuras}

Figura 2.1 - Exemplos de processos multicanal 32

Figura 2.2 - GCG proposto por Boyd (1950) 48

Figura 4.1 - Algoritmo utilizado na simulação para obtenção das medidas de desempenho individual

103

Figura 4.2 - Algoritmo utilizado na simulação para obtenção das medidas de desempenho conjunto

Figura 4.3 - Curva de valores de $\mathrm{NMA}_{1}$ para 2 canais e $\mathrm{NMA}_{0}=200(n=1)$

Figura 4.4 - Curva de valores de $\mathrm{NMA}_{1}$ para 3 canais e $\mathrm{NMA}_{0}=200(n=1)$

Figura 4.5 - Curva de valores de $\mathrm{NMA}_{1}$ para 15 canais e $\mathrm{NMA}_{0}=200(n=1)$

Figura 4.6 - Curva de valores de $\mathrm{NMA}_{1}$ para 20 canais e $\mathrm{NMA}_{0}=200(n=1)$

Figura 4.7 - Curva de valores de $\mathrm{NMA}_{1}$ para 2 canais e $\mathrm{NMA}_{0}=200(n=4)$

Figura 4.8 - Curva de valores de $\mathrm{NMA}_{1}$ para 3 canais e $\mathrm{NMA}_{0}=200(n=4)$

Figura 4.9 - Curva de valores de $\mathrm{NMA}_{1}$ para 15 canais e $\mathrm{NMA}_{0}=200(n=4)$

Figura 4.10 - Curva de valores de $\mathrm{NMA}_{1}$ para 20 canais e $\mathrm{NMA}_{0}=200(n=4)$

Figura 4.11 - Comparação entre os valores de $\mathrm{NMA}_{1}$ individuais e conjuntos para 3 canais considerando o valor de $\mathrm{NMA}_{0}$ igual a 200 amostras $(n=1)$

Figura 4.12 - Comparação entre os valores de $\mathrm{NMA}_{1}$ individuais e conjuntos para 20 canais considerando o valor de $\mathrm{NMA}_{0}$ igual a 200 amostras $(n=1)$

Figura 4.13 - Comparação entre os valores de $\mathrm{NMA}_{1}$ individuais e conjuntos para 3 canais considerando o valor de $\mathrm{NMA}_{0}$ igual a 200 amostras $(n=4)$

Figura 4.14 - Comparação entre os valores de $\mathrm{NMA}_{1}$ individuais e conjuntos para 20 canais considerando o valor de $\mathrm{NMA}_{0}$ igual a 200 amostras $(n=4)$

Figura 6.1 - Algoritmo utilizado na simulação para o período de aquecimento da estatística EWMA

Figura 6.2 - Algoritmo utilizado na simulação para obtenção das medidas de desempenho

Figura 6.3 - Curvas de valores de $\mathrm{NMA}_{0}$ para GCG EWMA DNB (3 canais)

Figura 6.4 - Curvas de valores de $\mathrm{NMA}_{0}$ para GCG EWMA DNB (20 canais)

Figura 6.5 - Curvas de valores de $\mathrm{NMA}_{0}$ para GC EWMA $\mathrm{R}_{\mathrm{t}}$ (3 canais) 
Figura 6.6 - Curvas de valores de $\mathrm{NMA}_{0}$ para GC EWMA $\mathrm{R}_{\mathrm{t}}$ (20 canais)

Figura 6.7 - Curvas de valores de $\mathrm{NMA}_{0}$ para GC MEWMA S ${ }^{2}(3$ canais)

Figura 6.8 - Curvas de valores de $\mathrm{NMA}_{0}$ para GC MEWMA $\mathrm{S}^{2}$ (20 canais)

Figura 6.9 - Curvas de valores de $\mathrm{NMA}_{1}$ para 15 canais, $\mathrm{NMA}_{0}=200$ e $\delta *=0,5(n=1)$

Figura 6.10 - Curvas de valores de $\mathrm{NMA}_{1}$ para 20 canais, $\mathrm{NMA}_{0}=200$ e $\delta *=0,5(n=1)$

Figura 6.11 - Curvas de valores de $\mathrm{NMA}_{1}$ para 15 canais, $\mathrm{NMA}_{0}=200$ e $\delta *=1,0(n=1)$

Figura 6.12 - Curvas de valores de $\mathrm{NMA}_{1}$ para 20 canais, $\mathrm{NMA}_{0}=200$ e $\delta *=1,0(n=1)$

Figura 6.13 - Curvas de valores de $\mathrm{NMA}_{1}$ para 15 canais, $\mathrm{NMA}_{0}=200$ e $\delta *=0,5(n=4)$

Figura 6.14 - Curvas de valores de $\mathrm{NMA}_{1}$ para 20 canais, $\mathrm{NMA}_{0}=200$ e $\delta *=0,5(n=4)$

Figura 6.15 - Curvas de valores de $\mathrm{NMA}_{1}$ para 15 canais, $\mathrm{NMA}_{0}=200$ e $\delta *=1,0 \quad(n=4)$

Figura 6.16 - Curvas de valores de $\mathrm{NMA}_{1}$ para 20 canais, $\mathrm{NMA}_{0}=200$ e $\delta *=1,0 \quad(n=4)$

Figura 7.1 - Algoritmo utilizado na simulação para o período de aquecimento individual da estatística EWMA

Figura 7.2 - Algoritmo utilizado na simulação para o período de aquecimento conjunto da estatística EWMA

179

Figura 7.3 - Algoritmo utilizado na simulação para obtenção das medidas de desempenho individual

Figura 7.4 - Algoritmo utilizado na simulação para obtenção das medidas de desempenho conjunto

Figura 7.5 - Curvas de valores de $\mathrm{NMA}_{0}$ para GCG EWMA MR DNB (3 canais)

Figura 7.6 - Curvas de valores de $\mathrm{NMA}_{0}$ para GCG EWMA MR DNB (20 canais)

Figura 7.7 - Curvas de valores de $\mathrm{NMA}_{0}$ para GCG EWMA $\ln \left(\mathrm{S}^{2}\right)$ (3 canais, $n=4$ )

Figura 7.8 - Curvas de valores de $\mathrm{NMA}_{0}$ para GCG EWMA $\ln \left(\mathrm{S}^{2}\right)$ (20 canais, $n=4$ )

Figura 7.9 - Comparação de desempenho entre as versões Shewhart e EWMA do GCG de DNB para NMA $\mathrm{N}_{0}=200(c=15, n=1)$

Figura 7.10 - Comparação de desempenho entre as versões Shewhart e EWMA do GC de $R_{t}$ para $\mathrm{NMA}_{0}=200(c=15, n=1)$

Figura 7.11 - Comparação de desempenho entre as versões Shewhart e EWMA do GC de $S^{2}$ para $\mathrm{NMA}_{0}=200(c=15, n=1)$

Figura 7.12 - Comparação de desempenho entre as versões Shewhart e EWMA do GCG de MR de DNB para $\mathrm{NMA}_{0}=200$ $(c=15, n=1)$

Figura 7.13 - Comparação de desempenho entre as versões Shewhart e EWMA do GCG de DNB para NMA $\mathrm{NM}_{0} 200(c=15, n=4)$ 
Figura 7.14 - Comparação de desempenho entre as versões Shewhart e EWMA do GC de $R_{\mathrm{t}}$ para $\mathrm{NMA}_{0}=200(c=15, n=4)$

Figura 7.15 - Comparação de desempenho entre as versões Shewhart e EWMA do GC de $S^{2}$ para $\mathrm{NMA}_{0}=200(c=15, n=4)$

Figura 7.16 - Comparação de desempenho entre o GCG de $S^{2}$ e o GCG EWMA de $\ln \left(\mathrm{S}^{2}\right)$ para $\mathrm{NMA}_{0}=200(c=15, n=4)$ 


\section{Lista de abreviaturas e símbolos}

$\alpha \quad$ probabilidade do erro tipo I

$\alpha_{\text {individual }} \quad$ probabilidade de alarme falso para cada canal individual

$\alpha_{\text {global }} \quad$ probabilidade de alarme falso global

A evento "canal afetado sinalizar"

AUO evento "qualquer canal sinalizar"

$A_{t} \quad$ variável aleatória que representa a variação do nível-base do processo. É a parcela de variação que é comum a todos os canais

ARL Average Run Length

1 vetor coluna de ordem $c \times 1$ de elementos iguais a 1

$b_{t} \quad$ valor real do nível-base

$\hat{b}_{t} \quad$ valor estimado do nível-base

$\beta \quad$ probabilidade do erro tipo II

c número de canais compondo o sistema

CEP Controle Estatístico de Processos

CUSUM Cumulative Sum

DNB Diferenças em relação ao Nível-Base

$\delta \quad$ deslocamento na média do processo

$\delta^{*} \quad$ valor de deslocamento na média do processo para o qual se obtém o projeto ótimo

$d_{2}, d_{3} \quad$ constantes determinadas pelo número total de canais no processo

$\varepsilon_{t} \quad$ ruído do processo

$e_{t j k} \quad$ variável aleatória que representa a componente individual de cada canal 


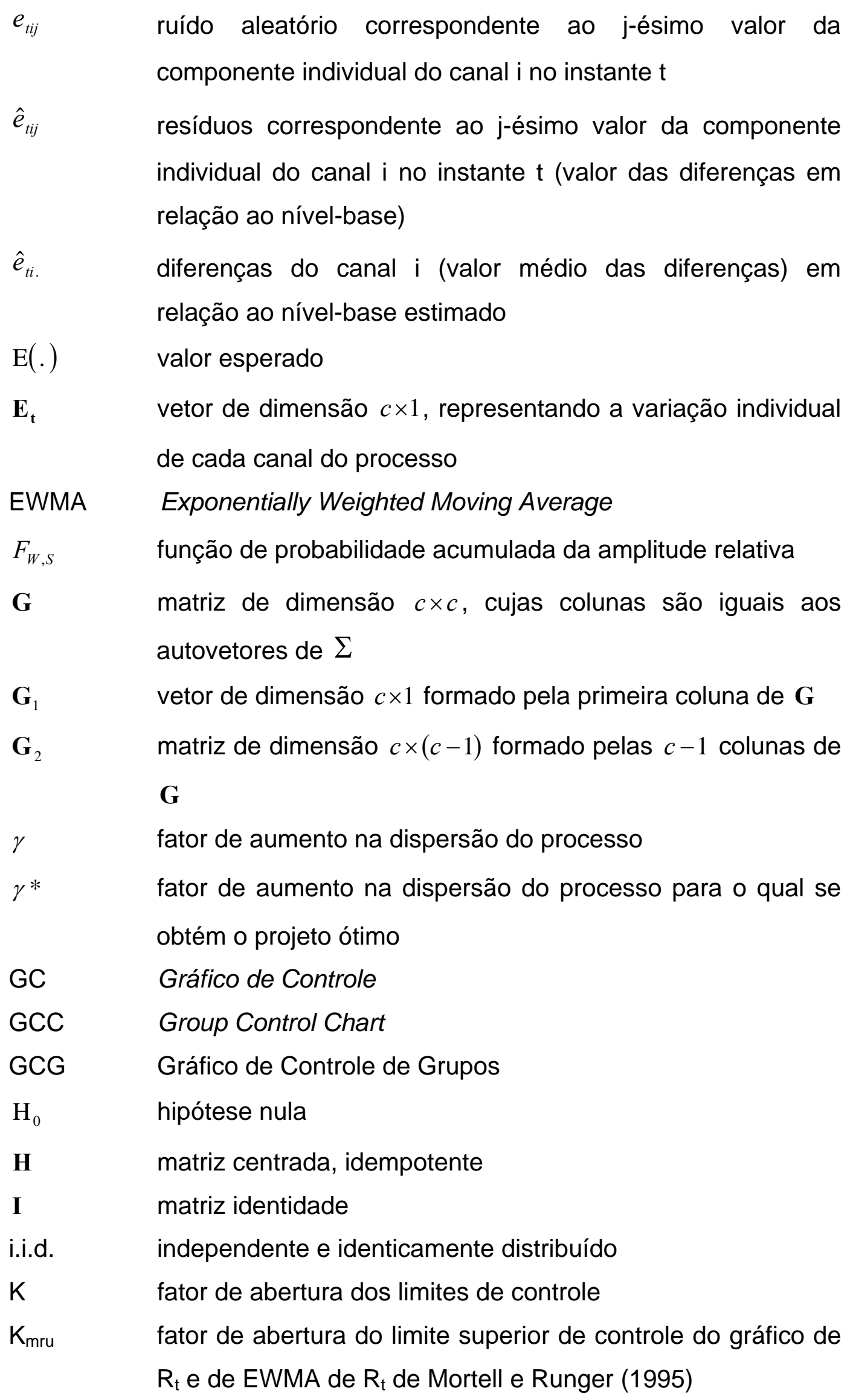


$\mathrm{K}_{\mathrm{dnb}} \quad$ fator de abertura dos limites de controle do gráfico das diferenças em relação ao nível-base de Barbosa (2008)

$\mathrm{K}_{\mathrm{MR}}$ fator de abertura do limite superior de controle do GCG de MR de DNB proposto

K Ins fator de abertura do limite superior de controle do GCG de EWMA de $\ln \left(S^{2}\right)$ proposto

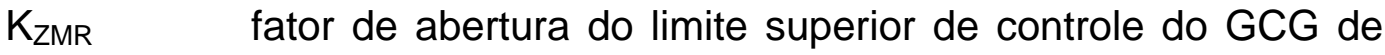
EWMA de MR DNB proposto

Kzdnb fator de abertura dos limites de controle do GCG de EWMA de DNB proposto

$\lambda \quad$ constante de amortecimento do modelo de EWMA

$\lambda * \quad$ valor da constante de amortecimento do modelo de EWMA que fornece o menor $\mathrm{NMA}_{1}$

LSCz limite superior de controle para o gráfico de controle de EWMA

$\mathrm{LSC}_{\mathrm{R}_{\mathrm{t}}} \quad$ limite superior de controle para o gráfico de controle de $R_{t}$ de Mortell e Runger (1995)

$\mathrm{LSC}_{\mathrm{Z}_{\mathrm{R}_{\mathrm{t}}}} \quad$ limite superior de controle para o gráfico de controle de EWMA de $R_{t}$ de Mortell e Runger (1995)

LSC $_{\text {RAM }}$ limite superior de controle para o gráfico de controle de $S^{2}$ de Runger, Alt e Montgomery (1996)

$\mathrm{LSC}_{\mathrm{GCGS}^{2}} \quad$ limite superior de controle para o $\mathrm{GCG}$ de $\mathrm{S}^{2}$ proposto

$\mathrm{LSC}_{\mathrm{GCGMR}}$ limite superior de controle para o GCG de MR proposto

$\mathrm{LSC}_{\mathrm{GCGZ}_{\mathrm{ln}}}$ limite superior de controle para o GCG de EWMA de $\ln \left(\mathrm{S}^{2}\right)$ proposto

LSC $_{\text {GCGZMR }}$ limite superior de controle para o GCG de EWMA de MR proposto

$\mathrm{LSC}_{\mathrm{Zd} n b} \quad$ limite superior de controle para o GCG de EWMA de DNB proposto 
$\mathrm{LICz}$ limite inferior de controle para o gráfico de controle de EWMA

$\mathrm{LIC}_{\mathrm{Zd} n b}$ limite inferior de controle para o GCG de EWMA de DNB proposto

$\ln \left(S^{2}\right) \quad$ variância amostral linearizada

$\mu \quad$ média do processo

$\mu_{0} \quad$ valor-alvo da média do processo

$\mathrm{MR}_{\mathrm{t}} \quad$ amplitude móvel no instante $\mathrm{t}$

MR DNB amplitude móvel das Diferenças em relação ao Nível-Base

MSP Multiple Stream Process

n tamanho de amostra por canal

NMA número médio de amostras até um sinal de descontrole no processo

$\mathrm{NMA}_{0} \quad$ número médio de amostras até um alarme falso

$\mathrm{NMA}_{1}$ número médio de amostras até um alarme verdadeiro, a partir do instante de ocorrência da causa especial de variação

$v$ parâmetro de não-centralidade da distribuição Qui-quadrado

$O \cap \bar{A} \quad$ evento "sinalização apenas por um canal não afetado"

PMC Processo Multicanal

r

Comprimento Significativo de Corrida

$\rho_{i j}$

coeficiente de correlação entre duas diferenças quaisquer $\hat{e}_{t i .}$ e $\hat{e}_{t j}$.

R amplitude amostral

$\mathrm{RL} \quad$ Run-Length

SDRL desvio-padrão de Run-Length

$S$ desvio-padrão amostral

$\mathrm{S}^{2} \quad$ variância amostral

$\sigma \quad$ desvio-padrão do processo

$\sigma_{0}^{2} \quad$ variância do processo quando está em controle

$\sigma_{1}^{2} \quad$ variância do processo quando está fora de controle 


\begin{tabular}{|c|c|}
\hline$\sigma_{a}$ & desvio-padrão da componente $A_{t}$ \\
\hline$\sigma_{e t i .}$ & desvio-padrão da componente $e_{\mathrm{ti}}$ \\
\hline$\sigma_{\mathrm{zt}}$ & desvio-padrão da estatística EWMA \\
\hline$\sigma\left(R_{t}\right)$ & desvio-padrão da amplitude amostral \\
\hline$\sigma\left(Z_{R_{t}}\right)$ & desvio-padrão da estatística EWMA da amplitude amostral \\
\hline$\sigma\left(Z_{\ln _{i}(t)}\right)$ & desvio-padrão da estatística EWMA de $\ln \left(\mathrm{S}^{2}\right)$ \\
\hline$\Sigma$ & matriz de covariâncias dos dados observados nos $c$ canais \\
\hline$T^{2}$ & estatística multivariada de Hotelling(1942) \\
\hline$\theta$ & conjunto de parâmetros do projeto do gráfico de controle \\
\hline$U_{1}$ & primeira componente principal \\
\hline$U_{2}$ & contém as $c-1$ componentes remanescentes \\
\hline $\operatorname{Var}()$. & variância \\
\hline VSSI & Variable Sample Size and Sampling Interval \\
\hline $\mathbf{X}_{t}$ & $\begin{array}{l}\text { vetor das observações de dimensão } c \times 1 \text { de todos os canais } \\
\text { no instante } t\end{array}$ \\
\hline$x_{t}$ & amostra retirada do processo no instante $t$ \\
\hline$x_{t j}$ & amostra retirada do canal $\mathrm{j}$ no instante $\mathrm{t}$ \\
\hline$x_{t i j}$ & j-ésima medida obtida no canal i no instante t \\
\hline$X_{t j k}$ & $\begin{array}{l}\text { variável aleatória que representa o valor esperado da k- } \\
\text { ésima observação contida no canal j no instante t }\end{array}$ \\
\hline$\overline{\mathrm{X}}$ & média amostral da variável $x$ \\
\hline $\mathrm{Z}_{0}$ & valor inicial da estatística de controle EWMA \\
\hline $\mathrm{Z}_{\mathrm{t}}$ & estatística de controle EWMA no instante t \\
\hline$Z_{\ln _{i}(t)}$ & $\begin{array}{l}\text { estatística de controle EWMA de } \ln \left(S^{2}\right) \text { do canal i no instante } \\
t\end{array}$ \\
\hline$Z_{M R_{i i}}$ & $\begin{array}{l}\text { estatística de controle EWMA de MR DNB do canal i no } \\
\text { instante } t\end{array}$ \\
\hline
\end{tabular}




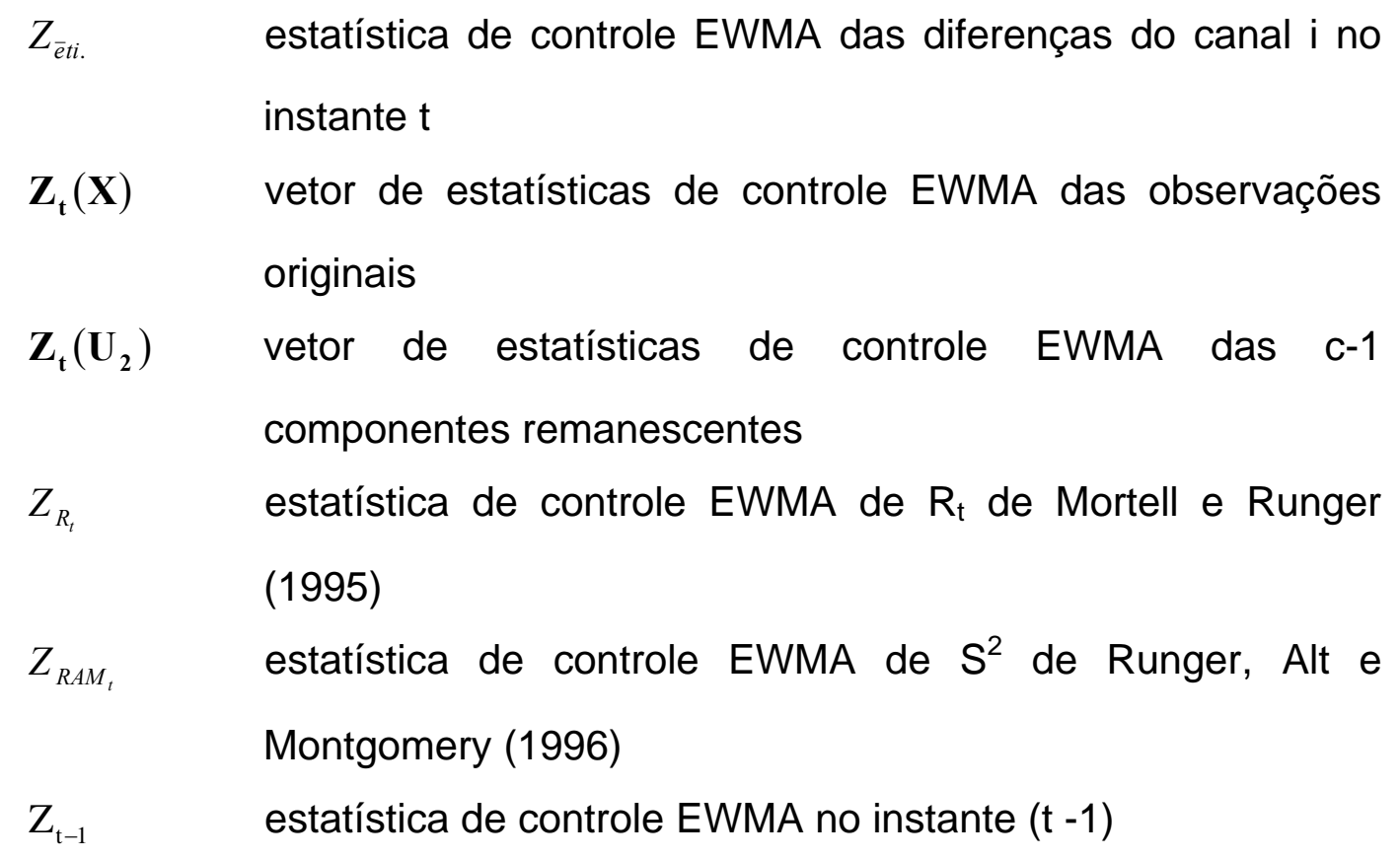

